\title{
ARTíCULO \\ Supervivencia de poslarvas de Litopenaeus vannamei sometidas a la prueba de estrés osmótico y su relación con el estado de muda
}

\author{
Survival of Litopenaeus vannamei postlarvae under osmotic stress \\ and its relation with the molt stage
}

\section{Elizabeth Burbano-Gallardoㅁ, Marco Antonio Imués-Figueroa ${ }^{1}$, Edgar Andrés Gonzalez-Legarda ${ }^{1}$, Luis Otavio Brito ${ }^{2}$, Alfredo Olivera Galvez ${ }^{3}$ y Luis Alejandro Vinatea Arana ${ }^{4}$}

\begin{abstract}
${ }^{1}$ Departamento de Recursos Hidrobiológicos, Facultad de Ciencias Pecuarias, Universidad de Nariño, San Juan de Pasto, Nariño, Colombia

${ }^{2}$ Departamento de Assistência Técnica e Extensão Rural, Instituto Agronômico de Pernambuco, Recife, Pernambuco, Brasil ${ }^{3}$ Laboratório de Maricultura Sustentável, Departamento de Pesca e Aquicultura, Universidade Federal Rural de Pernambuco, Recife, Pernambuco, Brasil

${ }^{4}$ Laboratório de Camarões Marinhos, Departamento de Aquicultura, Universidade Federal de Santa Catarina, Florianópolis, Santa Catarina, Brasil. vinatea@mbox1.ufsc.br
\end{abstract}

\begin{abstract}
This study was designed to evaluate the survival of shrimp postlarvae under osmotic stress and its relation with the molt stage. Postlarvae used were in stages PL7 to PL17. Osmotic stress was tested during intermolt stage (IM), initial premuda (PrMI) and final premuda (PrMF), determining its effect on survival. For this analysis the development of PLs gill, individuals weight and water quality parameters were taken into acount. Thirty three treatments in triplicate consisting of 2 factors, moult stages (IM, PrMF and PrMI) and age of postlarvae (PL17 to PL7). The results show significant differences for the interaction molt stage and age. The highest survival was found at the intermolt stage (74.12\%) from PL12.
\end{abstract}

Key words: Shrimp, salinity, age, intermolt, premuda

\begin{abstract}
Resumen.- Este estudio fue diseñado para evaluar la supervivencia de poslarvas de camarón sometidas a la prueba de estrés osmótico y su relación con el estado de muda. Se utilizaron poslarvas en los estadios de PL7 a PL17 y sometidas a prueba de estrés osmótico durante la etapa de intermuda (IM), premuda inicial (PrMI) y premuda final (PrMF), determinando su efecto por medio de la supervivencia. Para dicho análisis se tuvo en cuenta el desarrollo branquial de las poslarvas, el peso de los individuos y los parámetros de calidad de agua del cultivo. Se evaluaron 33 tratamientos en triplicado conformados por 2 factores: estadios de muda (IM, PrMI y PrMF) y edad (PL7 hasta PL17). Los resultados indican diferencias significativas para la interacción estadios de muda y edad. La supervivencia más alta se presentó en la etapa de intermuda $(74,12 \%)$ a partir de PL12.
\end{abstract}

Palabras clave: Camarón, salinidad, edad, intermuda, premuda

\section{INTRODUCCIÓN}

Durante los últimos años, con el desarrollo de la industria de cultivo del camarón, ha crecido la necesidad por parte de productores y compradores de semilla de garantizar la calidad de la misma, entendiéndose por calidad el buen desempeño de los animales durante el cultivo, reflejado en la resistencia a condiciones adversas y a enfermedades, altos porcentajes de supervivencia y buen crecimiento (Cuéllar-Anjel et al. 2010).

La supervivencia de larvas y poslarvas depende del estado de los progenitores, su condición nutricional, mantenimiento y condiciones de desove (Racotta et al. 2003, Palacios et al. 2004, Andriantahina et al. 2012). Para el caso de poslarvas, su estado fisiológico o calidad se evalúa según su movilidad, desarrollo morfológico, nivel de ramificación de las branquias, presencia de lípidos en el hepatopáncreas, amplitud del sexto segmento en relación con la longitud del intestino, color, apariencia, actividad, intervalo de tallas, duración de estadios larvarios y pruebas de estrés, entre otros (Cuéllar-Anjel et al. 2010). 
El camarón blanco Litopenaeus vannamei es una especie eurihalina que tolera un amplio intervalo de salinidades (5 a 45) (Briggs et al. 2005). Sin embargo, el punto isosmótico es de 27,7 (Romano \& Zeng 2012). Por otra parte, los intervalos de tolerancia a la salinidad y las condiciones óptimas de salinidad pueden variar durante el desarrollo ontogenético (Criales et al. 2011, ChongRobles et al. 2014). La supervivencia de poslarvas de camarón a la prueba de estrés por salinidad se debe principalmente a la capacidad osmorreguladora de los organismos y esto depende del grado de desarrollo de la poslarva, edad, fase de muda y su condición fisiológica (Álvarez et al. 2004).

Las pruebas de estrés permiten dilucidar la condición de las poslarvas, ya que son usadas como criterio de control de calidad en los laboratorios de producción de semilla y en los laboratorios de investigación (Arzola et al. 2013). La prueba de resistencia más utilizada consiste en someter las poslarvas a un estrés de baja salinidad, que consiste en una disminución abrupta de salinidad por tiempo determinado, permitiendo la posterior recuperación de los animales.

El ciclo de muda o ecdisis afecta ciertos aspectos de la biología de los crustáceos, incluyendo la morfología del animal, el metabolismo celular, la fisiología y su comportamiento (Bonilla-Gómez et al. 2012, Tumburu et al. 2012). Los camarones peneidos mudan en intervalos de pocos días o semanas. Su ciclo de muda está dividido en etapas: posmuda, intermuda, premuda y ecdisis (Corteel et al. 2012). Para determinar las etapas de muda se realiza la observación microscópica de los cambios morfológicos ocurridos en los urópodos de los camarones (Corteel et al. 2012). Cada proceso de muda que experimenta la larva implica en cambios morfológicos y fisiológicos, que se tornan más complejas a medida que el animal se desarrolla (Galindo et al. 2009). El conocimiento y habilidad para determinar las etapas del ciclo de muda en las poblaciones cultivadas es una herramienta importante de manejo para la transferencia de poslarvas desde los laboratorios hasta las camaroneras (Olin \& Fast 1992). La mayoría de los criterios son visuales, tales como el comportamiento natatorio. Es indispensable disponer de un método fiable y fácil para evaluar la calidad de las poslarvas producidas en laboratorio. En este sentido, el presente estudio tuvo como objetivo mejorar el proceso de aplicación de pruebas de estrés en poslarvas de Litopenaeus vannamei evaluando la relación existente entre la etapa de muda, edad y el estrés osmótico.

\section{MATERIALES Y MÉTODOS}

El experimento se realizó en el área de precría de Laboratorio de Camarones Marinos (LCM) de la Universidad Federal de Santa Catarina, Brasil, donde un lote de reproductores se dispuso en un estanque con volumen de $50 \mathrm{~m}^{3}$. Se aplicó un diseño completamente aleatorizado con muestreo y arreglo factorial 3 x 11 para 33 tratamientos con 3 repeticiones cada uno, en donde el factor 1 (F1) fue el estadio de muda (intermuda, premuda inicial y premuda final) y el factor 2 (F2) la edad de las poslarvas (PL7 a PL17).

Se utilizaron poslarvas de L. vannamei provenientes de los estanques de maduración internos, de $9 \mathrm{~m}^{2}$ con $0,50 \mathrm{~m}$ de profundidad, salinidad de 34 , temperatura de $28^{\circ} \mathrm{C}$, fotoperiodo natural (13 h luz: $11 \mathrm{~h}$ oscuridad), en sistema de cultivo en biofloc según Avnimelech (1999): relación carbono/nitrógeno de 20:1. Igualmente, en los reproductores fue practicada la ablación ocular unilateral (solo en hembras), relación hembras/machos de 1:1 (con peso individual de $35 \pm 7,2 \mathrm{~g}$ ) y la alimentación con el $20 \%$ del peso corporal diario con calamares, poliquetos y pescado fresco. Las poslarvas resultantes fueron alimentadas con ración Potimar Active, con $35 \%$ de proteína bruta. A partir de un estanque de $50 \mathrm{~m}^{3}$ se recolectaron las muestras de poslarvas desde la edad de PL7 a PL17. El estanque fue equipado con sistema de aireación, calentador de $6000 \mathrm{~kW}$ y tuberías de abastecimiento de agua marina y continental.

Se trabajó con 3300 poslarvas, las que se mezclaron aleatoriamente en el lote de producción utilizado, sometiéndolas a la prueba de estrés osmótico en cada uno de los periodos de muda establecidos. Para dicha prueba se trabajó con 330 individuos para identificar su desarrollo branquial y 660 para determinar los estadios de muda. Las poslarvas fueron depositadas en tubos Falcón con alcohol al 10\% y posteriormente se realizó el análisis con la ayuda de un microscopio óptico binocular (Zeiss, Axiostar plus, Gottingen) en 10X y 40X, observando la morfología de los urópodos para la clasificación de los estadios de muda de acuerdo a Robertson et al. (1987) y Chan et al. (1988). En el primer estadio de muda, denominado intermuda (IM), se observó una sola matriz celular o epidermis, seguido de un segundo estadio denominado premuda inicial (PrMI), el cual se caracterizó por un leve desprendimiento de la cutícula de la matriz celular sin la total separación de la epidermis. La última etapa de muda, denominada premuda final (PrMF), se identificó por la total separación de la matriz celular, observándose nuevas setas en desarrollo. Estas 
características fueron determinadas en cada uno de los estadios poslarvas evaluados (PL7 a PL17).

Se tomaron diariamente muestras de poslarvas durante la fase de evaluación. A partir de las muestras recolectadas se determinó su estado general en relación al estadio, periodo de muda, desarrollo branquial, sometimiento a pruebas de estrés y peso seco. Del mismo modo, se determinó que las poslarvas estuvieran libres de enfermedades, observando la limpieza de las branquias y, en el caso de presentar necrosis o bacterias filamentosas, las poslarvas fueron descartadas.

Posteriormente, se tomaron 3 muestras de 10 poslarvas de cada estadio, y se observó bajo microscopio óptico binocular (Zeiss, Axiostar plus, Gottingen, Germany) el desarrollo de lóbulos branquiales y sus ramificaciones. Se contó la cantidad de divisiones de cada filamento branquial y se determinó el índice de desarrollo branquial de la población según Artiles et al. (1999), aplicando la fórmula:

$$
\begin{aligned}
& \mathrm{DBI}=\mathrm{RF} / \text { Número de filamentos } \\
& \mathrm{DBP}=\mathrm{DBI} / \text { Número de poslarvas }
\end{aligned}
$$

donde, $\mathrm{DBI}=$ Desarrollo branquial de cada individuo; $\mathrm{RF}=$ número de divisiones de cada filamento; y $\mathrm{DBP}=$ desarrollo branquial de la población analizada.

Para la prueba de estrés osmótico se tomaron, por medio de una pipeta de $100 \mathrm{ml}, 3$ muestras de 100 poslarvas del lote de producción, las cuales se mantuvieron en salinidad de 35. Las poslarvas fueron contadas y depositadas por triplicado en vasos de precipitado de $1 \mathrm{~L}$ con agua de salinidad 0, sometiéndolas de esta forma a un cambio brusco de salinidad. Las poslarvas se mantuvieron en estas condiciones por $30 \mathrm{~min}$, regresándose posteriormente a la salinidad inicial (35) durante otros $30 \mathrm{~min}$. Al término de este tiempo se contó el número de poslarvas sobrevivientes en cada recipiente expresado como porcentaje del número inicial. Las larvas sobrevivientes a la prueba no se devolvieron al estanque de cultivo, siendo automáticamente descartadas.

Diariamente, se realizó la medición de los parámetros de calidad de agua del cultivo de poslarvas, oxígeno disuelto y temperatura (sonda YSI 550A), $\mathrm{pH}$ (pH metro, YSI pH100), salinidad (refractómetro, Zhifong, FG-102) y transparencia (disco de Secchi). Estos parámetros fueron determinados 2 veces al día, a las 7:00 y 17:00 h.

Se tomaron del cultivo 3 submuestras de 50 poslarvas, tanto al inicio como al final del experimento, para estimar la tasa de crecimiento específico. Las submuestras se depositaron en un crisol con papel aluminio previamente deshidratado durante $24 \mathrm{~h}$ con la ayuda de una estufa de secado (DeLeo, DL-AFDT, Brasil, Porto Alegre) a temperatura constante de $60^{\circ} \mathrm{C}$. Posteriormente, se determinó el peso seco empleando balanza analítica. La Tasa de crecimiento específica (TEC) se determinó de acuerdo a Heinsbroek (1990) con la fórmula:

$$
\mathrm{TEC}=100 \mathrm{x}(\ln \mathrm{pf}-\ln \mathrm{pi}) / \mathrm{T})
$$

donde $\mathrm{pf}=$ peso final; $\mathrm{pi}=$ peso inicial $\mathrm{y} \mathrm{T}=$ tiempo.

Esto se realizó para cada una de las edades y en cada estadio de muda definidos. Para el análisis estadístico de los datos se utilizó un generador de números aleatorios (Excel, Microsoft) para aleatorizar la distribución de los tratamientos. Para la comparación de los valores de supervivencia de las poslarvas ante la prueba de estrés, atendiendo a la edad y a las etapas de muda, los datos fueron consignados como porcentajes y transformados mediante una función arco seno. La homogeneidad de varianzas se evaluó mediante la prueba de Bartlett y el supuesto de independencia mediante la prueba de DurbinWatson. Se utilizó un test de ANOVA multifactorial $(P<$ $0,05)$ para determinar el efecto de los factores sobre la supervivencia de las poslarvas sometidas al estrés osmótico. En los casos en los cuales se encontraron diferencias significativas entre los grupos se realizó una prueba de comparación múltiple de Tukey $(P<0,05)$. Todas las pruebas se efectuaron utilizando el software Statgraphics Centurion 2010 Microsoft.

\section{Resultados}

Los parámetros físicos y químicos de temperatura, oxígeno disuelto, salinidad, $\mathrm{pH}$ y transparencia medidos diariamente durante el periodo de estudio fueron considerados normales a lo largo de todo el experimento (Tabla 1).

En la Tabla 2 se muestra el desarrollo branquial de las poslarvas evaluadas y se indica el conteo de filamentos branquiales en cada una de las edades, observándose un desarrollo inicial de 4 filamentos para PL7 y la presencia de 14 filamentos para PL17. De igual forma, se determinaron los valores del índice de desarrollo branquial individual (DBI) con un valor inicial de 0,50 para PL7 y 0,88 para PL11, y el índice de desarrollo poblacional (DBP) con 0,017 para PL7 y 0,029 para PL11. A partir de PL12 en adelante no se calcularon estos índices puesto que la cantidad de ramificaciones en estas edades son muy abundantes y no permiten su conteo. 
Tabla 1. Parámetros físicos y químicos medidos en el estanque de producción de poslarvas / Physico-chemical parameters measured in tank postlarvae production

\begin{tabular}{lr}
\hline \multicolumn{1}{c}{ Parámetros } & Promedio y D.E \\
\hline Temperatura $\left({ }^{\circ} \mathrm{C}\right)$ & $29,30 \pm 0,93$ \\
Oxígeno $\left(\mathrm{mg} \mathrm{L}^{-1}\right)$ & $5,73 \pm 0,25$ \\
Salinidad & $35 \pm 0,00$ \\
pH & $8,18 \pm 0,16$ \\
Transparencia $(\mathrm{cm})$ & $37,18 \pm 2,79$ \\
\hline
\end{tabular}

Tabla 2. Desarrollo branquial de las poslarvas y conteo de filamentos branquiales para cada una de las edades / Gill development of postlarvae and counting indicated gill filaments in each of the ages

\begin{tabular}{lcccc}
\hline Edad & $\begin{array}{c}\mathrm{N}^{\circ} \\
\text { Filamentos }\end{array}$ & RF & DBI & DBP \\
\hline PL7 & 4 & 2 & 0,50 & 0,017 \\
PL8 & 5 & 2 & 0,40 & 0,013 \\
PL9 & 6 & 4 & 0,67 & 0,022 \\
PL10 & 7 & 6 & 0,86 & 0,029 \\
PL11 & 8 & 7 & 0,88 & 0,029 \\
PL12 & 9 & Altamente ramificadas & & \\
PL13 & 10 & Altamente ramificadas & & \\
PL14 & 11 & Altamente ramificadas & & \\
PL15 & 12 & Altamente ramificadas & & \\
PL16 & 13 & Altamente ramificadas & & \\
PL17 & 14 & Altamente ramificadas & & \\
\hline
\end{tabular}

DBI (Desarrollo branquial de cada individuo) $=\mathrm{RF}$ (Número de divisiones de cada filamento) / Número de filamentos

DBP (Desarrollo branquial de la población analizada $)=$ DBI / Número de poslarvas
Los resultados de supervivencia obtenidos de la prueba de estrés osmótico realizada en las diferentes etapas de muda y edad de las poslarvas presentaron diferencias estadísticamente significativas para ambos factores y sus interacciones $(P=0,0028)$ (Tabla 3$)$. El promedio de supervivencia en la etapa de intermuda fue del $74,12 \%$, mientras que en la etapa de premuda inicial alcanzó el 60,24\% y en la etapa de premuda final llegó solo a 46,73\%, indicando que la supervivencia más alta se presentó en la etapa de intermuda.

En la Tabla 4 se presenta la supervivencia de las poslarvas a la prueba de estrés osmótico en cada una de las edades evaluadas con las comparaciones entre todos los pares de tratamientos. El análisis mostró diferencias significativas entre grupos de tratamientos (interacción entre muda y edad). En las etapas de premuda para las edades de PL7 a PL17 se presentaron valores bajos de supervivencia en relación a la fase de intermuda. Los mejores valores de supervivencia (91 al 100\%) se obtuvieron la fase de intermuda, en las edades de PL15 a PL17.

Durante el periodo de estudio las poslarvas presentaron una tasa de crecimiento del $41 \%$, lo que significa que los animales incrementaron diariamente su masa corporal viéndose reflejado en la variable peso, presentando un promedio de peso seco inicial de $0,18 \pm$ $0,027 \mathrm{mg}$ en estadio de PL7 y peso seco final de $15,42 \pm$ 0,267 mg en PL 17.

Tabla 3. Interacción entre factores para porcentaje de supervivencia de poslarvas sometidas a prueba de estrés osmótico durante sus etapas de muda y edad / Interaction factors for percent survival of postlarvae tested osmotic stress during molting stages and age

\begin{tabular}{lccccc}
\hline \multicolumn{1}{c}{ Fuente } & $\begin{array}{c}\text { Suma de } \\
\text { Cuadrados }\end{array}$ & Gl & $\begin{array}{c}\text { Cuadrado } \\
\text { Medio }\end{array}$ & F & $P$ \\
\hline Tratamientos & 19,5913 & 32 & 0,612227 & 64,06 & $0,0000^{*}$ \\
A: Factor1 & 2,42484 & 2 & 1,21242 & 126,86 & $0,0000^{*}$ \\
B: Factor2 & 16,6876 & 10 & 1,66876 & 174,60 & $0,0000^{*}$ \\
Interacciones AB & 0,47884 & 20 & 0,023942 & 2,51 & $0,0028^{*}$ \\
Error experimental & 0,630789 & 66 & 0,0095574 & & \\
Total & 20,222 & 98 & & & \\
\hline
\end{tabular}

*Existen diferencias significativas $(P<0,05)$ 
Tabla 4. Interacción de la edad y el estado de muda sobre la supervivencia de poslarvas de $L$. vannamei sometidas a la prueba de estrés osmótico / Interaction of age and molting on the survival of $L$. vannamei poslarvae under osmotic stress test

\begin{tabular}{|c|c|c|c|}
\hline Tratamiento & Muda-Edad & $\begin{array}{c}\text { Media } \\
\%\end{array}$ & $\begin{array}{c}\text { Grupos } \\
\text { homogéneos }\end{array}$ \\
\hline 23 & PrMF-PL7 & 0,00 & $\mathrm{X}$ \\
\hline 12 & PrMI-PL7 & 1,67 & XX \\
\hline 24 & PrMF-PL8 & 4,67 & XX \\
\hline 1 & IM-PL7 & 9,33 & XX \\
\hline 25 & PrMF-PL9 & 12,00 & $\mathrm{X}$ \\
\hline 13 & PrMI-PL8 & 12,00 & XX \\
\hline 2 & IM-PL8 & 14,30 & $X X$ \\
\hline 14 & PrMI-PL9 & 15,00 & XX \\
\hline 26 & PrMF-PL10 & 18,30 & XX \\
\hline 15 & PrMI-PL10 & 38,00 & XX \\
\hline 27 & PrMF-PL11 & 48,30 & $\mathrm{XX}$ \\
\hline 28 & PrMF-PL12 & 53,60 & XX \\
\hline 3 & IM-PL9 & 56,00 & XXX \\
\hline 30 & PrMF-PL14 & 61,00 & XXXX \\
\hline 29 & PrMF-PL13 & 62,60 & XXXX \\
\hline 4 & IM-PL10 & 64,00 & XXXX \\
\hline 16 & PrMI-PL11 & 72,60 & XXXX \\
\hline 32 & PrMF-PL16 & 72,00 & XXXX \\
\hline 19 & PrMI-PL14 & 77,30 & XXXXX \\
\hline 18 & PrMI-PL13 & 82,30 & XXXXX \\
\hline 17 & PrMI-PL12 & 83,30 & XXXXX \\
\hline 31 & PrMF-PL15 & 85,00 & XXXXX \\
\hline 21 & PrMI-PL16 & 89,00 & XXXXX \\
\hline 5 & IM-PL11 & 91,30 & XXXXX \\
\hline 20 & PrMI-PL15 & 92,30 & XXXXX \\
\hline 8 & IM-PL14 & 94,30 & XXXXX \\
\hline 10 & IM-PL16 & 94,60 & XXXX \\
\hline 9 & IM-PL15 & 96,00 & XXXX \\
\hline 33 & PrMF-PL17 & 96,30 & XXXX \\
\hline 7 & IM-PL13 & 96,60 & XXXX \\
\hline 6 & IM-PL12 & 98,60 & XXX \\
\hline 22 & PrMI-PL17 & 99,00 & XX \\
\hline 11 & IM-PL17 & 100,00 & X \\
\hline
\end{tabular}

La Prueba de Tukey, mediante letras diferentes, indica que hay diferencias estadísticamente significativas

\section{Discusión}

Los parámetros físicos y químicos del agua (temperatura, oxígeno disuelto, salinidad, $\mathrm{pH}$ y transparencia) medidos diariamente durante el periodo de estudio resultaron dentro de los valores recomendados para el cultivo de camarón marino (Van Wyk \& Scarpa 1999).

Las supervivencias obtenidas en la prueba de estrés osmótico se presentaron más altas en la etapa de intermuda $(74,12 \%)$. En relación en edades la supervivencia fue mayor en el estadio de PL17 (98,44\%). Estos resultados sugieren que la tolerancia al estrés osmótico se incrementa a medida que aumenta la edad de las larvas, pero con una tendencia mucho mayor cuando las poslarvas se encuentran en fase de intermuda (IM), siendo más baja en la fase de premuda final (PrMF). La sensibilidad al estrés osmótico se incrementa a medida que avanza el ciclo de muda y la misma disminuye con la edad.

Probablemente el ciclo de muda y las diferentes concentraciones de salinidad influyen en la absorción de nutrientes de los camarones, los cuales se muestran más débiles en las fases de premuda inicial (PrMI) y premuda final (PrMF), puesto que existen alteraciones en el metabolismo de los aminoácidos (Shinji et al. 2012a), en la digestibilidad aparente de carbohidratos y lípidos de los alimentos (Gucic et al. 2013), en la capacidad osmótica, hemocianina, glucosa, glucógeno en el hepatopáncreas (Galindo et al. 2009) y la capacidad osmótica y proteína total (Bonilla-Gómez et al. 2012). Las actividades enzimáticas digestivas están fuertemente afectadas por el ciclo de la muda en $L$. stylirostris como una respuesta fisiológica a las exigencias metabólicas de energía y nutrientes (Casillas-Hernández et al. 2002).

Ceccaldi (1997) señala que esta conducta puede deberse al hecho de que en el proceso de despojarse del exoesqueleto algunas estructuras como la boca, el esófago y parte del estómago dejan de ser funcionales. Estas estructuras poseen una capa de quitina que es continuación de las capas externas, y que se desprenden junto con el antiguo exoesqueleto impidiendo que se sigan realizando las funciones normales de prensión, tránsito y molienda del alimento. Por otro lado, durante el proceso de muda las branquias quedan parcialmente inutilizadas por la misma causa y las poslarvas solo vuelven a respirar normalmente después de adquirir la rigidez necesaria. Según Vega et al. (1999) este proceso de desprendimiento de las capas quitinosas del sistema digestivo comienza durante la premuda final, en la cual el animal no puede comer desde ese momento, por lo que pasa a utilizar los lípidos y carbohidratos de reserva para llevar con éxito las etapas subsecuentes de la muda como la construcción del nuevo exoesqueleto.

En el análisis para el factor 2 (edad), el desarrollo branquial determinó que a mayor edad mayor es la presencia de filamentos branquiales. De igual forma, el índice de desarrollo branquial individual (DBI) y poblacional (DBP) resultaron ser directamente proporcionales a la edad de las poslarvas. Es posible sugerir que, dado el papel de las branquias en la osmoregulación, al menos parte del aumento de la supervivencia puede deberse a un mayor desarrollo 
branquial en larvas de mayor edad. Sin embargo, no es el único factor porque las etapas del ciclo de muda también están influidas por la supervivencia.

La interacción entre los 2 factores (etapas de muda y edades) aumentaron a medida que aumentó la edad de las poslarvas, por lo que se determinó que los mejores tratamientos para realizar dichas pruebas de estrés que demuestren su calidad deberían ser realizadas en la etapa de intermuda y en las edades de PL15 a PL17. La sobrevivencia a la prueba de estrés en poslarvas de camarón es considerada buena cuando los valores son superiores a 75\% (ABCC 2012). Valores obtenidos a partir de PL11 en IM, PL12 en PRMI y PL17 en PrMF dieron los mayores resultados de sobrevivencia cuando fueron analizados bajo el efecto de la interacción edad y estadio de muda.

Arzola et al. (2013) observaron valores entre 94,4 y $99,8 \%$ de supervivencia de poslarvas (PL 12) durante las diferentes combinaciones de salinidad $(5,15,25,35$ y 45) y temperatura $\left(15,20,25,30\right.$ y $\left.35^{\circ} \mathrm{C}\right)$, probablemente debido al desarrollo integral de los arcos branquias en la fase de PL 12, que están directamente ligados al proceso de osmorregulación. Esta tolerancia a las variaciones de salinidad ocurre cuando la capacidad osmoregulatoria de esta especie sigue aumentando gradualmente, al menos hasta PL35. Según Criales et al. (2011) entre la PL35 y la PL55 (Farfantepenaeus duorarum) no hubo diferencias y las larvas ya habrían alcanzado en esta etapa la máxima capacidad osmoregulatoria.

Según Rees et al. (1994), a edades tempranas la capacidad de osmorregulación de las poslarvas es deficiente debido a que las estructuras que realizan dicha función (branquias) no están completamente ramificadas y su área de intercambio no es suficiente para compensar un choque osmótico o todavía no pueden realizar un intercambio iónico eficiente. Otros estudios demostraron que factores como la hormona hiperglicemica (Shinji et al. 2012b) y los perfiles metabólicos de la hemolinfa (Jagabattula et al. 2013) se modifican de acuerdo a las diferentes salinidades en las cuales los camarones fueron sometidos y pueden influir en su desarrollo.

Los valores de peso medio de las poslarvas varían de acuerdo con las densidades de cultivo, origen de los reproductores, calidad de agua y del alimento proporcionado. Los resultados promedio finales de peso seco 15,42 $\pm 0,267 \mathrm{mg}$ en PL 17 en este trabajo indicaron que los camarones fueron bien alimentados y este factor no influyó en las sobrevivencias obtenidas. En resumen, los resultados de esta investigación pueden contribuir en la mejora de los protocolos usados para determinar la calidad de las poslarvas de L. vannamei que son comercializadas por laboratorio de producción. Dentro de los protocolos para determinar la calidad de las poslarvas se debe tener en cuenta el ciclo de la muda del camarón, ya que al presentarse bajas supervivencias en la prueba de estrés no necesariamente significa que el lote de sea de mala calidad.

Se concluye que, dada la interacción existente entre edad y etapa del ciclo de muda, las pruebas de estrés osmótico deberían hacerse solo con animales en intermuda y en poslarvas no menores al estadio 12 para individuos cultivados en agua de salinidad de 35 y temperatura de $29^{\circ} \mathrm{C}$.

\section{Agradecimientos}

Los autores desean agradecer a Consejo Nacional de Desarrollo Científico y Tecnológico (CNPq). Los autores también desean agradecer a Felipe do Nascimento Vieira, José Luiz Pedreira Mouriño, Carlos Manoel do Espírito Santo (Laboratorio de Camarones Marinos, Departamento de Acuicultura, Universidad Federal de Santa Catarina, Brasil) por sus contribuciones en este estudio. Se agradece también a los árbitros anónimos por sus valiosas sugerencias. Luis Vinatea fue becario de investigación del CNPq.

\section{LiTERATURA CITADA}

ABCC. 2013. Boas práticas de manejo e biossegurança para a carcinicultura marinha nacional, 58 pp. Associação Brasileira de Criadores de Camarão, Natal.

Álvarez AL, IS Racotta, O Arjona \& E Palacios. 2004. Salinity stress test as a predictor of survival during growout in Pacific white shrimp (Litopenaeus vannamei). Aquaculture 237: 237-249.

Andriantahina F, X Liu, H Huang, J Xiang \& C Yang. 2012. Comparison of reproductive performance and offspring quality of domesticated Pacific white shrimp, Litopenaeus vannamei. Aquaculture 324-325: 194-200.

Artiles M, B Jaime \& L Pérez-Jar. 1999. Evaluación de la calidad de poslarvas de camarón blanco Penaeus schmittii mediante diferentes pruebas de estrés. Revista de Investigaciones Marinas 20(1-3): 93-102.

Arzola GJ, V Pablo-Piña, S Mario-Nieves \& J MaríaMedina. 2013. Supervivencia de poslarvas de camarón blanco Litopenaeus vannamei a diferentes salinidades y temperaturas Revista MVZ Córdoba 18(Supl): 3618-3625. 
Avnimelech Y. 1999. Carbon/nitrogen ratio as a control element in aquaculture systems. Aquaculture 176(3-4): 227-235.

Bonilla-Gómez JS, X Chiappa-Carrara, C Galindo, G Jeronimo, G Cuzon \& G Gaxiola. 2012. Physiological and biochemical changes of wild and cultivated juvenile pink shrimp Farfantepenaeus duorarum (Crustacea: Penaeidae) during molt cycle. Journal of Crustacean Biology 32(4): 597-606

Briggs M, S Funge-Smith, RP Subasinghe \& M Phillips. 2005. Introductions and movement of two penaeid shrimp species in Asia and the Pacific. FAO Fisheries Technical Paper 476: 1-63.

Casillas-Hernández R, F Magallón, G Portillo, O Carrillo, H Nolasco \& F Vega-Villasante. 2002. La actividad de proteasa, amilasa y lipasa durante los estadios de muda del camarón azul Litopenaeus stylirostris. Revista de Investigaciones Marinas 23(1): 35-40.

Ceccaldi H. 1997. Anatomy and physiology of the digestive system. In: Abramo L, D Conklin \& D Akiyama (eds). Crustacean nutrition, advances in world aquaculture, pp. 261-291. The World Aquaculture Society, Baton Rouge.

Chan SM, M Susan, SM Rankin \& LL Keeley. 1988. Characterization of the molt stages in Penaeus vannamei: Setogenesis and hemolymph levels of total protein, ecdysteroids, and glucose. The Biological Bulletin 175: 185192.

Chong-Robles J, G Charmantier, V Boulo, J LizárragaValdéz, LM Enríquez-Paredes, I Giffard-Mena. 2014. Osmoregulation pattern and salinity tolerance of the white shrimp Litopenaeus vannamei (Boone, 1931) during postembryonic development. Aquaculture 422-423: 261-267.

Corteel M, JJ Dantas-Lima, M Wille, V Alday-Sanz, MB Pensaert, P Sorgeloos \& HJ Nauwynck. 2012. Moult cycle of laboratory-raised Penaeus (Litopenaeus) vannamei and P. monodon. Aquaculture International 20: 13-18.

Criales MM, IC Zink, JÁ Browder \& TL Jackson. 2011. The effect of acclimation salinity and age on the salinity tolerance of pink shrimp poslarvae. Journal of Experimental Marine Biology and Ecology 409: 283-289

Cuéllar-Anjel J, C Lara, V Morales, AD Gracia \& OG Suárez. 2010. Manual de buenas prácticas de manejo para el cultivo del camarón blanco Penaeus vannamei, 132 pp. OIRSA-OSPESCA, Panamá.

Galindo C, G Gaxiola, G Cuzon \& X Chiappa-Carrara. 2009. Physiological and biochemical variations during the molt cycle in juvenile Litopenaeus vannamei under laboratory conditions. Journal of Crustacean Biology 29(4): 544-549.
Gucic M, E Cortés-Jacinto, R Civera-Cerecedo, D RicqueMarie \& LR Martínez-Córdova. 2013. Apparent carbohydrate and lipid digestibility of feeds for whiteleg shrimp, Litopenaeus vannamei (Decapoda: Penaeidae), cultivated at different salinities. Revista de Biologia Tropical 61(3): 1201-1213.

Jagabattula SD, K Ambasankar, R Rajendran, V Rajaram \& M Muralidhar. 2013. Effect of abiotic salinity stress on haemolymph metabolic profiles in cultured tiger shrimp Penaeus monodon. International Journal of Bioresource and Stress Management 4(2): 339-343.

Heinsbroek L. 1990. Growth and feeding of fish, 93 pp. Department of Fish Culture and Fisheries, Wageningen Agricultural University, Wageningen.

Olin P \& W Fast. 1992. Penaeid PL harvest, transport, acclimation and stocking. In: Fast AW \& LJ Lester (eds). Marine shrimp culture: Principles and practices, pp. 301320. Elsevier Science Publishers, Amsterdam.

Palacios E, A Bonilla, A Pérez, IS Racotta \& R Civera. 2004. Influence of highly unsaturated fatty acids on the responses of white shrimp (Litopenaeus vannamei) poslarvae to low salinity. Journal of Experimental Marine Biology and Ecology 299: 201-215.

Racotta IS, E Palacios \& AM Ibarra. 2003. Shrimp larval quality in relation to broodstock condition. Aquaculture 227: 107-130.

Rees J, K Cure, S Piyatiratitivorakul, P Sorgeloos \& P Menasveta. 1994. Highly unsaturated fatty acid requirements of Penaeus monodon poslarvae an experimental approach based on Artemia enrichment. Aquaculture 122(3): 193-207.

Robertson L, W Bray, J Leung-Truillo \& A Lawrence. 1987. Practical molt staging of Penaeus setiferus and Penaeus stylirostris. Journal of the World Aquaculture Society 18: 180-185.

Romano N \& C Zeng. 2012. Osmoregulation in decapod crustaceans: implications to aquaculture productivity, methods for potential improvement and interactions with elevated ammonia exposure. Aquaculture 334-337: 12-23.

Shinji J, T Okutsu, V Jayasankar, S Jasmani \& MN Wilder. 2012a. Metabolism of amino acids during hyposmotic adaptation in the whiteleg shrimp, Litopenaeus vannamei. Amino Acids 43(5): 1945-1954.

Shinji J, BJ Kang, T Okutsu, K Banzai, T Ohira, N Tsutsui \& MN Wilder. 2012b. Changes in crustacean hyperglycemic hormones in Pacific whiteleg shrimp Litopenaeus vannamei subjected to air-exposure and lowsalinity stresses. Fisheries Science 78: 833-840. 
Tumburu L, EF Shepard, AE Strand \& CL Browdy. 2012.

Effects of endosulfan exposure and Taura Syndrome Virus infection on the survival and molting of the marine penaeid shrimp, Litopenaeus vannamei. Chemosphere 86: 912-918.

Vega F, I Fernández, R Preciado, M Oliva, D Tovar \& H Nolasco. 1999. The activity of the digestive enzymes during the molting stages of the arched swimming Callinectes arcuatus Ordway, 1863 (Crustacea: Decapoda: Portunidae). Bulletin of Marine Science 65(1): 1-9.
Van Wyk P \& J Scarpa. 1999. Water quality requirements and management. In: Van Wyk P, M Davis-Hodgkins, R Laramore, KL Main, J Mountain \& J Scarpa (eds). Farming marine shrimp in recirculating freshwater systems, pp. 141162. Florida Department of Agriculture and Consumer Services / Harbor Branch Oceanic Institute, Florida.

Recibido el 29 de diciembre 2014 y aceptado el 13 de mayo de 2015

Editor: Claudia Bustos D. 